\title{
EFFECT OF ACCELERATED AGING ON THE COLOR STABILITY AND BOND STRENGTH OF TWO TYPES OF GLASS-BASED CERAMICS: AN IN-VITRO STUDY
}

\author{
Mahmoud S. Alsayed*, Khaled M. Haggag ${ }^{* *}$, Husain R. Mohammed ${ }^{* * *}$
}

\begin{abstract}
Objective: The objective of the present study was to investigate the effect of accelerated aging on the color stability and bond strength of two types of glass-based ceramics. Materials and methods: A total of 42 disk-shaped specimens were fabricated from two ceramic materials; IPS e.max CAD and Vita Suprinity; 21 for each ceramic material, with dimensions of $14 \mathrm{~mm}$ length $\times 12 \mathrm{~mm}$ width $\times 1 \mathrm{~mm}$ thickness were obtained and crystalized according to the manufacture instructions. They were then divided randomly into 2 groups: the first group was 14 discs, 7 from each ceramic material for the initial color measurement using a spectrophotometer. The second group consisted of 28 disc-shaped specimens, 14 for each ceramic material, cemented to their corresponding composite resin disk under static load using dual-cured resin cement. Shear bond strength test for 7 specimens from each group was done by compressive load applied at composite-ceramic interface using the universal testing machine traveling at cross-head speed of 0.5 $\mathrm{mm} / \mathrm{min}$. The failure load was recorded in Newton. The aging process was done by the use of special weathering machine called Environmental Incubator Model. The discs that were artificially aged underwent the same color and bond strength measurements as before the artificial aging process. Results: Artificial accelerated aging had a statistically significant effect on the color stability of tested groups. Vita Suprinity recorded higher color difference $(\Delta \mathrm{E})$ mean value $(2.52 \pm 0.63)$ than E.max group $(1.61 \pm 0.48)$. There was no statistically significant difference in the shear bond strength test of two ceramic materials. Conclusions: Both ceramic materials performance was clinically acceptable regarding their color stability. E.max CAD showed significant better color stability than Vita Suprinity. Both ceramic materials had similar bond strength values.
\end{abstract}

KEY WORDS: Glass based ceramics, Accelerated aging, color stability, Bond strength

\section{INTRODUCTION}

One of the major challenges for modern dentistry is the achievement of a perfect and optimal optical properties of natural teeth with artificial restorative materials. Among these materials, dental ceramics can closely reproduce the appearance of the natural teeth, having an optical behavior very close to that of dental hard tissues ${ }^{(1-7)}$.

The clinical success and durability of indirect fixed restorations are affected by the cementation procedures. Adhesive bonding systems are introduced in dental practice not only to improve the retention between the restoration and the tooth structure but also to achieve better aesthetic results and maintain the highest ceramic strength ${ }^{(8-12)}$.

As the glass-based ceramics are formed of mixture of glass matrix containing crystalline phases, the use of hydrofluoric acid combined with silane coupling agent could achieve high bond strength between the restoration and the luting cement ${ }^{(8-12)}$. Lithium disilicate ceramic can provide the highest esthetic demands with exceptional userfriendliness. Recently, a new group of machinable ceramics has been introduced for computer aided design /computer aided manufacture (CAD/CAM)

\footnotetext{
* Demonstrator, Crown and Bridge Department Faculty of Dental Medicine, Al-Azhar University, Cairo.

** Professor, Crown and Bridge Department Faculty of Dental Medicine, Al-Azhar University, Cairo.

*** Lecturer, Crown and Bridge Department Faculty of Dental Medicine, Al-Azhar University, Cairo.
} 
techniques; zirconia-reinforced lithium silicate ceramics. According to the manufacturers, these materials are characterized by high mechanical properties and are comparable with the clinically well-proven lithium disilicate glass ceramics ${ }^{(13)}$.

Artificial accelerated aging is a laboratory method used to simulate environmental destructive capacity and predict the relative durability of materials exposed to a hostile oral environment by simulating the chemical and physical conditions to which restorative materials are expected to face in the oral cavity conditions ${ }^{(14,15)}$.

The null hypothesis of this study was that there will be no difference in color stability and bond strength between the test groups after accelerated aging.

\section{MATERIALS AND METHODS}

Two materials were used in this study, IPS e.max CAD and Vita Suprinity. A total of 42 disc-shaped specimens, 21 for each ceramic material, with the dimensions of $14 \mathrm{~mm}$ length $\times 12 \mathrm{~mm}$ width $\times 1 \mathrm{~mm}$ thickness were obtained using micro-saw (Isomet 4000 micro saw, Buehler, USA). The thickness of each disc was verified by digital caliper with 0.002 accuracy (Alpha-Tools Digital Caliper, Oakland, NJ, USA). All specimens were crystalized according to the manufacturer instructions, then divided randomly into 2 groups. The first group were 14 discs, 7 from each material to be used for color stability measurement. The second group consisted of 28 disc-shaped specimens, 14 for each ceramic. They were used for shear bond strength test measurement so that the present study was conducted in to two parts:

\section{Color stability measurement:}

The initial color of 14 ceramic discs ( 7 from each material) was measured using a portable reflective spectrophotometer (X-Rite, model RM200QC, Neu-Isenburg, Germany). The aperture size was set to $4 \mathrm{~mm}$ and the specimens were exactly aligned with the device. A white background was selected based on ISO standers (ISO 7491) and measurements were made according to the CIE L*a*b* color space relative to the CIE standard illuminant D65 ${ }^{(14)}$.

\section{Shear bond strength measurement test:}

A specially designed split brass mold was machine milled and used to obtain total of 28 circular composite (3M EZ 250, shade A2, USA) disks with dimensions of $5 \mathrm{~mm}$ diameter $\times 3 \mathrm{~mm}$ thickness.

The hydrofluoric acid etching followed by silaine application (Dent bond, ITENA, France) was performed according to the manufacturer instructions for the predetermined superior surface of the ceramic disks and the composite disks were then cemented to their corresponding ceramic ones under static load using dual-cured resin cement (Bifix QM, Resin-Based Adhesive Luting System, Voco America, Inc). Fourteen specimens, 7 of each ceramic material were subjected to shear bond strength test using a universal testing machine (Figure 1).

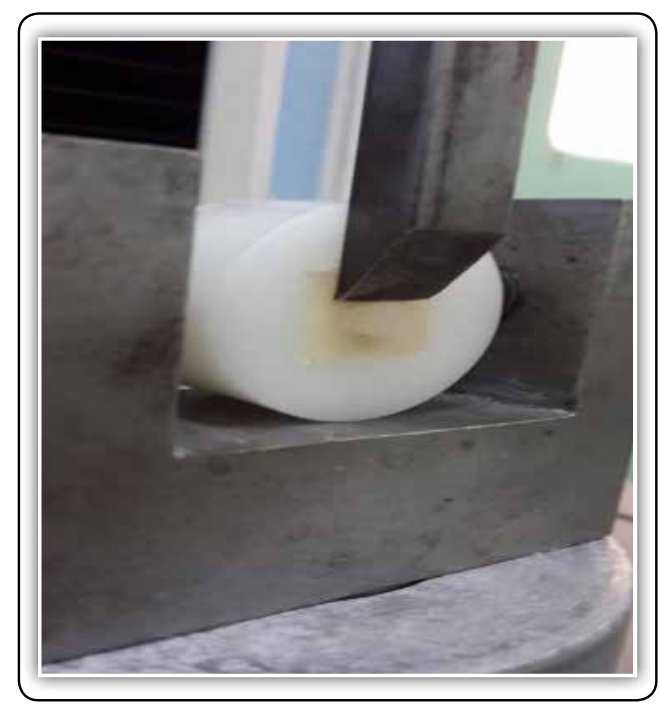

FIG (1) Shear bond strength testing machine

A circular interface shear test was designed to evaluate the bond strength. All specimens 
were individually and horizontally mounted on a computer-controlled testing machine (Model 3345; Instron Industrial Products, Norwood, USA) with a load cell of $5 \mathrm{kN}$ and data were recorded using computer software (Bluehill Lite; Instron Instruments). Specimens were secured to the lower fixed compartment of testing machine by tightening screws through Teflon custom made housing device with central cavity into which the ceramic plate fit (dimensions; $14 \times 12 \times 1 \mathrm{~mm}$ ). Shear bond strength test was done by compressive mode of load applied at composite-ceramic interface using a mono-beveled chisel shaped metallic rod attached to the upper movable compartment of testing machine traveling at cross-head speed of $0.5 \mathrm{~mm} / \mathrm{min}$. The failure load was recorded in Newton.

The aging process was done for the remaining specimens ( 7 specimens from each material) in addition to the disks that already used for initial color measurement ( 7 disks from each material). The use of special weathering machine called Environmental Incubator Model (Jeio Tech TEMI 300. Korea). All specimens were placed in equipment shelf and exposed to 9 UV-B light source with radiation of $280 / 320 \mathrm{~nm}$, at distance of $50 \mathrm{~nm}$, in condensation chamber. The program was set for 4 hours of UV-B exposure at $55^{\circ} \mathrm{C}$, and 4 hours of condensation at $5^{\circ} \mathrm{C}$ with $100 \%$ humidity for 300 hours, equivalent to 1 year of clinical service ${ }^{(14)}$.

Artificially aged specimens underwent the same color and bond strength measurements as for before the artificial aging process. Disks were evaluated for color stability by calculating the color difference of the specimens before and after accelerated aging sing the CIElab $\Delta \mathrm{E}$ equation. Data obtained from all testes were collected, tabulated, and subjected to statistical analysis.

\section{RESULTS}

All data were fed to the computer and analyzed using IBM SPSS software package version 20.0. (Armonk, NY: IBM Corp). The KolmogorovSmirnov test was used to verify the normality of distribution.

Quantitative data were described using range (minimum and maximum), mean, standard deviation and median. Significance of the obtained results was judged at the 5\% level. Mann-Whitney test was used for abnormally distributed quantitative variables, to compare between the two groups. Wilcoxon-signed-ranks test was used for abnormally distributed quantitative variables, to compare between two periods.

There was a significant statistical difference in the color change between the studied groups, the greater amount of color change was found in vita suprinity group with $\Delta \mathrm{E}$ value of $(2.52 \pm 0.63)$ greater than e.max group which have $\Delta \mathrm{E}$ value of $(1.61 \pm 0.48)(p<0.005)$. There was no significant difference in the shear bond strength of both types of ceramic materials before and after artificial accelerated aging (Table (1) and Figure (2)).

TABLE (1) Descriptive statistics using Mann-Whitney test for color $(\Delta \mathrm{E})$ and shear bond strength values (MPa)

\begin{tabular}{|c|c|c|c|c|c|c|c|c|c|}
\hline Material & Aging & Mean & SD & Max & Med & Min & $\mathrm{U}$ & $\mathrm{P}$ value & \\
\hline \multirow{2}{*}{ 它苍 } & IPS e.max CAD & & 1.61 & 0.48 & 2.18 & 1.66 & 0.91 & \multirow{2}{*}{3.0} & \multirow{2}{*}{0.047} \\
\hline & VITA SUPRINITY & & 2.52 & 0.63 & 3.38 & 2.67 & 1.71 & & \\
\hline \multirow{4}{*}{ 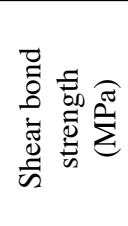 } & \multirow{2}{*}{ IPS e.max CAD } & Without aging & 9.57 & 2.09 & 13.23 & 8.50 & 8.29 & \multirow{2}{*}{0.535} & \multirow{2}{*}{0.593} \\
\hline & & After aging & 8.25 & 2.74 & 12.10 & 6.57 & 6.14 & & \\
\hline & \multirow{2}{*}{ VITA SURINITY } & Without aging & 11.12 & 2.0 & 14.14 & 11.01 & 9.03 & \multirow{2}{*}{1.069} & \multirow{2}{*}{0.285} \\
\hline & & After aging & 10.50 & 3.69 & 16.01 & 9.63 & 7.06 & & \\
\hline
\end{tabular}




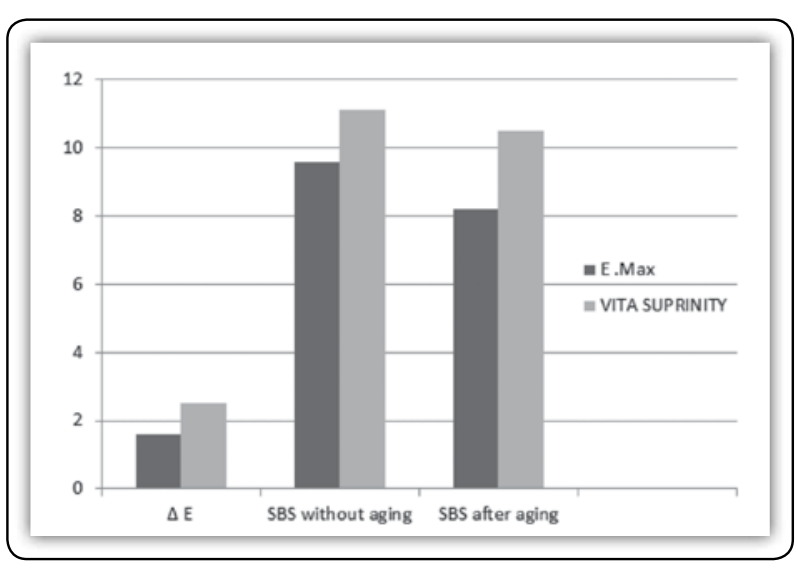

FIG (1) Diagrammatic chart representing the mean color difference $\Delta \mathrm{E}$ of studied groups after accelerated aging, mean shear bond strength of the studied group before and after artificial accelerated aging (AAA).

\section{Failure mode}

In this study, IPS e.max exhibit predominately type I adhesive failure (71.5\%), and less Type III mixed failure $(28.5 \%)$ with no existence of type II cohesive failure $(0 \%)$.

Vita Suprinity group exhibited predominately type II cohesive failure (57.5\%), followed by Type III mixed failure $(42.5 \%)$ with no existence of type I adhesive failure $(0 \%)$.

\section{DISCUSSION}

\section{Color stability}

Although the color change is one of the primary factors for failure of esthetic restorations, little is known about color changes in ceramic restorations over time, as investigators focused on the mechanical properties of the material. However, the adverse conditions of the oral environment may cause changes in the physical properties of the material with subsequent color change ${ }^{(2,3)}$.

In the present study the ceramic discs were sawed by the micro saw to be of $1 \mathrm{~mm}$ thickness $14 \mathrm{~mm}$ length and $12 \mathrm{~mm}$ width of both IPS e.max CAD and Vita Suprinity. The disk-shaped specimens were used in the present study for color evaluation.
As in several previous studies ${ }^{(4-7)}$, disks are the most appropriate way to measure the light translucency and color of the tested specimens, since important factors such as size and surface quality can be standardized ${ }^{(16)}$.

The first part of the null hypothesis that artificial accelerated aging will have no significant effect on the color stability of the two ceramic materials was rejected. With regards to color stability of tested ceramic materials, the perceptibility level set in this study was $2 \Delta \mathrm{E}$ units, whereas the clinical acceptability level was set to $3.7 \Delta \mathrm{E}$ units as concluded by many studies ${ }^{(15,17,18)}$.

Vita Suprinity group showed the greatest amount of color change with $\Delta \mathrm{E}$ value of $2.52 \pm 0.63$ greater than e.max CAD group which had $\Delta \mathrm{E}^{*}$ value of $1.61 \pm 0.48(\mathrm{p}<0.005)$. According to that, $\Delta \mathrm{E}^{*}$ value e.max CAD $(<2)$ is non-perceptible and clinically acceptable. However, Vita Suprinity $\Delta \mathrm{E}(>2)$ is perceptible but still clinically acceptable. The color change observed in Vita Suprinity group may be due to breakdown of the metal oxide content of the colorants added to the ceramic itself. The breakdown of metal oxides under ultraviolet radiation is followed by peroxide compound formation that would likely change the color of the shaded ceramic material ${ }^{(19)}$. The measurements were performed on specimens with simple shape (discs) and the effect of variables related to the production of the abutment-shaped restoration was not investigated in the present study.

\section{Shear bond strength}

The unglazed ceramic surfaces were used in this study because in the clinical situation the internal surface of the restoration remains unglazed. Theoretically, unglazed surfaces exhibit greater irregularities and micromechanical interlocking and thus higher bond strength compared to glazed surfaces ${ }^{(20)}$. Composite discs of (3mm thickness and $5 \mathrm{~mm}$ diameter) were bonded to ceramic discs. Hydrofluoric acid was applied as a surface treatment 
strategy in the present investigation and improves the bond strength. Etching with hydrofluoric acid could be considered an effective modality for glass ceramics as it dissolves the glassy phase of acidsusceptible ceramics, producing suitable surface texture for bonding and facilitating the penetration of resin into ceramic ${ }^{(9)}$

Macro shear test was used due to its simplicity and practicability. Ozyoney (21) discussed the accuracy and clinical relevance of the different testing methods and showed that the shear bond strength is the most common testing method.

The second part of null hypothesis that will be no difference in the shear bond strength of both types of ceramic materials after artificial accelerated aging was accepted. The results of the present study showed that there was no significant different in the shear bond strength of two ceramic materials. The resin bond to silica-based ceramics, like lithium disilicates, is well documented. Preferred bonding methods are hydrofluoric acid-etching and subsequent silane treatments ${ }^{(10)}$.

In the present study, most of the failures in e.max $\mathrm{CAD}$ were adhesive in nature. This is in agreement with the results of recent studies by Özcan et al ${ }^{(22)}$, Gürol et al ${ }^{(12)}$ and El Damanhoury et al ${ }^{(23)}$. The bond between ceramic and resin cement is influenced by several factors such as temperature changes, saliva, diet, chewing force and other habits in a complex oral environment. We did not employ fatigue cycle in this study because of the diversity of the treatment groups and the controversial results of previous studies regarding the effect of these procedures on shear bond strength.

\section{CONCLUSIONS}

Within the limitations of the present in-vitro study, it can be concluded that both ceramic materials' color stability was clinically acceptable, althuough IPS e.max CAD had higher color stability than Vita Suprinity. Both ceramic materials had similar bond strength values.

\section{REFERENCES}

1. Hammad IA. Interpreter repeatability of shade selections with two shade guides. J Prosthet Dent. 2003;89(1):50-3

2. Abdelbary O, Wahsh M, Sherif A, Salah T. Effect of accelerated aging on translucency of monolithic zirconia. Future Dental Journal 2016; 2:65-69.

3. Paravina RD, Ontiveros JC, Powers JM. Accelerated aging effects on color and translucency of bleaching-shade composites. J Esthet Restor Dent. 2004;16(2):117-26.

4. Dozic' A, Kleverlaan CJ, Meegdes M, Zel JV, FeilzerAJ. The influence of porcelain layer thickness on the final shade of ceramic Restorations. J Prosthet Dent 2003; 90:563-70

5. Lim HN, Yu B, Jin Ik, Lim JK, Lee YK. Correlations between Spectro radiometric and spectrophotometric colors of all-ceramic materials. Dent Mater 2010; 26:1052-8.

6. Ahn SJand Lee YK. Difference in the translucency of allceramics by the illuminant. Dent Mater 2008; 24:1539-44.

7. Lim HN, Yu B, and Lee YK. Spectro radiometric and Spectrophotometric translucency of ceramic materials. J Prosthet Dent 2010; 104:239-46.

8. Menees TS, Lawson NC, Beck PR, Burgess JO. Influence of particle abrasion or hydrofluoric acid etching on lithium disilicate flexural strength. J Prosthet Dent. 2014; 112:1164-70.

9. Tian T, Tsoi JK, Matinlinna JP, Burrow MF. Review Aspects of bonding between resin luting cements and glass ceramic materials. Dent Mater. 2014; 7:47-62.

10. Chen L, Suh B. Bonding of resin materials to all-ceramics: A Review. Current Research in Dentistry 2012; 3: 7-17.

11. Ahrari F, Boruziniat A, Mohammad H, and Alirezaei M The effect of surface treatment with a fractional carbon dioxide laser on shear bond strength of resin cement to a lithium disilicate-based ceramic. Dent Res J. 2017 ;3: 195-202.

12. Gürol O, Funda Y, Dilek T, Nuran O, Mustafa O. Shear Bond Strength of Composite Resin Cements to Ceramics. Marmara Dental Journal (2013) 2: 61-66.

13. Shaymaa E, Amr M. Mechanical properties of zirconia lithium silicate glass ceramic. Den Mater. 2016; 32(7): 908-14.

14. Pires-de-Souza Fde C, Casemiro LA, Garcia Lda F, Cruvinel DR. Color stability of dental ceramics submitted to accelerated aging after repeated firings. J Prosthet Dent 2009; 101:13-8 
15. Paravina RD, Ontiveros JC, Powers JM. Accelerated aging effects on color and translucency of bleaching-shade composites. J Esthet Restor Dent 2004; 16:117-26.

16. Schmitter M, Mueller D, and Rues S. Chipping behavior of all-ceramic crowns with zirconia framework and CAD/ CAM manufactured veneer. J Dent 2012; 40:154-62.

17. Johnston WM, Kao EC. Assessment of appearance match by visual observation and clinical colorimetry. J Dent Res 1989; 68:819-22.

18. Sakai M. The optical effect of zirconia background, ceramic thickness, and cement on all-ceramic material (Master Thesis). Chic, Illinois: University of Illinois, 2012.

19. Turgut $\mathrm{S}$, and Bagis B. Color stability of laminate veneers: an in vitro study. J Dent 2011; 39:57-64.
20. Heintze SD, Albrecht T, Cavalleri A, Steiner M. A new method to test the fracture probability of all-ceramic crowns with a dual-axis chewing simulator. Dent Mater 2011; 27:10-19

21. Ozyoney G, Yanıkoğlu F, Tağtekin D, Ozyoney1 N, Oksüz M. Shear Bond Strength of Composite Resin Cements to Ceramics. Marmara Dental Journal .2013; 2: 61-66.

22. Ozcan M, Vallittu P. Effect of surface conditioning methods on the bond strength of luting cement to ceramics. Dent Mater. 2003; 19:725-31.

23. El Damanhoury H, Gaintazopoulou M. Self-etching ceramic primer versus hydrofluoric acid etching: Etching efficacy and bonding performance. J Prosthodont Res. 2018;62(1):75-83. 